\title{
A STOCHASTIC FORECAST MODEL FOR JAPAN'S POPULATION
}

\author{
By \\ Yoichi Okita \\ Professor, National Graduate Institute for Policy Studies \\ 7-22-1 Roppongi, Minato-ku, Tokyo 106-8677 Japan \\ okita-yo@grips.ac.jp \\ Wade D. Pfau (corresponding author) \\ Associate Professor, National Graduate Institute for Policy Studies \\ 7-22-1 Roppongi, Minato-ku, Tokyo 106-8677 Japan \\ wpfau@grips.ac.jp \\ and \\ Giang Thanh Long \\ Lecturer, National Economics University (NEU) \\ 207 Giai Phong Street, Hai Ba Trung District, Hanoi 10000, Vietnam \\ longgt@neu.edu.vn
}

May 2009

\begin{abstract}
Obtaining appropriate forecasts for the future population is a vital component of public policy analysis for issues ranging from government budgets to pension systems. Traditionally, demographic forecasters rely on a deterministic approach with various scenarios informed by expert opinion. This approach has been widely criticized, and we apply an alternative stochastic modeling framework that can provide a probability distribution for forecasts of the Japanese population. We find the potential for much greater variability in the future demographic situation for Japan than implied by existing deterministic forecasts. This demands greater flexibility from policy makers when confronting population aging issues.
\end{abstract}

JEL Classification: J1, C53

Keywords: stochastic population forecasts, Japan, Lee-Carter method 


\section{INTRODUCTION}

An important component of public policy analysis for issues ranging from government budgets to pension systems is to have appropriate forecasts for the future population. Particularly in Japan, population aging is expected to progress at a rapid rate, which is creating many difficult challenges for public policy as there will be fewer workingage people to support the consumption of the elderly. Fertility rates and mortality rates have been falling drastically in Japan, creating a situation in which Japanese people experience some of the longest lifespans and lowest fertility rates of any country. Already, the total population of Japan is decreasing. What is not clear, though, is how these trends will evolve in the future. In assessing the details of future government and pension finances, policy makers are confounded by the uncertainties of forecasting a multitude of demographic trends. The accuracy of population forecasts are important to determine the ratio between beneficiaries and contributors for the pension system, for instance, because it is clear that decisions made now will have long-term effects on the solvency of pensions and public debt. For Japan's case, the concern is particularly important, as Kunieda (2002) explains that Japan's pension system is poorly designed as future generations are being exploited to pay for the generous pension benefits of current generations. The degree of this exploitation depends on how the population age structure evolves. While it is impossible to precisely forecast long-term population growth, forecasters can at least strive to provide a realistic range of possibilities.

Traditionally, demographic forecasters rely on a deterministic approach informed by expert opinion, with three potential scenarios that are meant to provide a range of possible future outcomes. Three scenarios may be made separately for fertility and mortality, and then the scenarios will be combined to forecast the total population. To obtain the widest range for the total population, high fertility will be combined with low mortality (long lives) for the high population case, and low fertility will be combined with high mortality for the low population case. In contrast, when population forecasts are used for long-term pension forecasts, the three scenarios usually include a low-cost ("optimistic") alternative with relatively few elderly people combining high fertility and high mortality, an intermediate-cost ("best guess”) alternative, and a high-cost ("pessimistic”) alternative with low fertility, low mortality, and relatively more elderly. This deterministic approach does not provide any sense of the likelihood with which these future scenarios may occur, such as why fertility and mortality would be perfectly correlated, and it combines the assumptions in overly rigid ways, such as not allowing for the possibility of cyclical baby booms and baby busts. The 
probabilities of falling in the high-low ranges will be different across each variable, such that when they are all put together, any sense of probability will be lost. In other words, it is not clear what the alternative scenarios really mean. This point of view is summarized well by the Advisory Council on Social Security (1991):

Further work is necessary to define the conceptual framework for the current low- and high-cost projections. Although theoretically they represent a collection of extreme values for each of the variables, how they should be interpreted is not obvious. For example, possible interpretations include: absolute bounds on what could possibly happen, confidence intervals, illustrative alternative projections, and sensitivity analyses.

This deterministic forecasting approach also places too much reliance on its point estimates for forecasts without considering the possible dispersion of results, and so it can produce forecasts that completely miss subsequent trends. Yamashige (2007) demonstrates this clear tendency for forecasts of the total fertility rate in Japan made by the National Institute of Population and Social Security Research (NIPSSR) at five year intervals between 1976 and 2001. As Japanese fertility experienced a continuous decline from over 2 in 1970 to about 1.3 in the early 2000s, forecasters repeatedly predicted that the fertility declines were temporary and the trend would reverse itself and fertility would increase in the following years, and they were repeatedly wrong.

Another problem with this three scenario approach, as explained in Lee (1999), is that it can produce misleading results for comparisons of population subgroups, such as the proportion of the population that is elderly, or the old-age dependency ratio (the ratio of population aged $65+$ to the population aged 15-64). When high fertility is combined with low mortality, there are more working-age people and more elderly people, and when low fertility is combined with high mortality, there are less working-age and elderly people. Consequently, the differences between the extreme scenarios will be artificially small.

An important example of this deterministic forecasting approach for Japan, which we will use as a point of comparison for our results, is Kaneko et al. (2008), which presents the population projections of NIPSSR in December 2006 based on the 2005 Census. These forecasts are for 2006 through 2105, though they mainly focus on forecasting through 2055 and make further extrapolations assuming mortality and fertility rates stay their 2055 levels for later years. Their forecasts provide three scenarios (low, medium, and high) for each of fertility and mortality. Their scenario leading to the highest population combines high fertility rates with low mortality rates, while their scenario producing the lowest population combines low fertility rates with high mortality rates. Along with the medium-medium 
forecast, these are the three scenarios we will compare to our total population forecasts. For considering population subgroups such as the proportion of elderly, the highest proportion results from the combination of low fertility rates and low mortality rates, while the lowest proportion of elderly results from the combination of high fertility and mortality rates. As such, these additional forecasts will be considered as well when relevant.

Researchers in recent years have been taking steps toward correcting some of the problems associated with deterministic forecasts by creating stochastic forecasting models that better deal with the uncertainties. These researchers are interested to use more rigorous time series econometric techniques to create stochastic forecasts which include probability distributions to provide a better understanding of the likelihood for various outcomes. Unlike deterministic forecasts, stochastic forecasts can be used to estimate a probability distribution for potential outcomes and to better capture their volatility. These models are usually parsimonious, estimating a few parameters for a reduced form time series model explaining past trends, rather than attempting to build a structural model or incorporate expert opinion to explain demographic change. This approach fits a model for the past trends and volatility of key variables, and then creates simulations of future outcomes using these estimated characteristics.

Key developments in this area include Lee and Carter (1992), which provides a methodology for making a stochastic forecast of mortality rates in the United States using a singular value decomposition with only a few parameters. Lee (1993) expands the method to apply to the less predictable fertility rates by also incorporating additional constraints on the potential fluctuations in the total fertility rate, and Lee and Tuljapurkar (1994) combines the fertility and mortality forecasts using a cohort component model to make a stochastic forecast for the United States population. Lee (1999) provides further justifications for using the stochastic approach for population forecasting. For other countries, Hyndman and Booth (2008) produce a forecast for Australia, and Keilman, Pham, and Hetland (2002) examine the case of Norway.

Often these population forecasts are made for the purposes of analyzing long-term government finances and in particular the sustainability of pension systems. For instance, Lee and Tuljapurkar (2001) and Tuljapurkar (2006) extend stochastic population forecasts to consider long-term fiscal planning for government budgets. For pension finances, stochastic forecasts have been used by government agencies in the United States, including the Congressional Budget Office (2005) which updates a report first made in 2001, and the Social Security Administration Board of Trustees (2003). Burdick and Manchester (2003) 
compare the results of these government modeling efforts, as well as the results of Lee, Anderson, and Tuljapurkar (2003), a report commissioned by the Social Security Administration. More recently, Sabelhaus and Topoleski (2007) extend the stochastic model of the Congressional Budget Office to consider reforms which are tied to realized economic and demographic outcomes. As well, Pfau (2003) applies this stochastic forecasting approach to hypothetical workers to understand more about the implications of a variety of Social Security reforms in the United States.

For other countries, Giang and Pfau (2009) use stochastic forecasts to study the future evolution of Vietnam's pension system, Fehr and Habermann (2004) analyze the sustainability of the German pension system under demographic uncertainty during 2001-2050, Børlum (2004) finds also that demographic uncertainty will have substantial influences on the long-term benefit levels of different participating cohorts in the Danish pension system, Kitamura, Tsurubuchi, and Nakashima (2006) use the Asset-Liability Model (ALM) with stochastic simulation to analyze the Japanese Public Employees' Pension Scheme, and Pfau and Atisophon (2009) use stochastic forecasts to study the implications of adapting a defined-contribution pension system in Thailand. Most relevant to this study, Suzuki, Yuda, and Kawasaki (2003) use a stochastic forecast for fertility, in order to make a stochastic forecast for Japan's population, but otherwise keep the other assumptions matched to the NIPPSR deterministic forecasts, to study the implications of demographic forecasts on pension finances in Japan. In particular, their study uses deterministic forecasts for mortality. Their approach to forecasting fertility is to apply past estimates of fertility rate functions using economic variables, but this approach also predicts a rebound in fertility rates and a much more gradual decrease in the total population.

In this paper, we consider a more comprehensive stochastic model for Japan's population. Specifically, we develop stochastic forecasts for both mortality and fertility rates in Japan, and combine these forecasts to understand the possible evolution of Japan's population for different age groups. This allows us to examine a range of possibilities for such important factors as the total fertility rate, life expectancies, total population, and the proportion of elderly people in the population. Creating a probability distribution allows for a more careful consideration of different scenarios, and also provides information about whether existing deterministic forecasts show enough variability to capture possible future trends. We find that the 95 percent confidence intervals for most of our measures tend to be noticeably wider than the extreme deterministic forecasts, which indicates that the future 
demographic situation in Japan is potentially more volatile than can be assumed from existing forecasting approaches.

\section{METHODOLOGY}

Our basic method for projecting the population is the cohort component method, which uses forecasts for mortality, fertility, and migration to make year-by-year modifications to a base population through the use of a Leslie matrix. Constructing population forecasts is accomplished with an iterative procedure, starting with a base. To forecast the population in subsequent years, we use the population of the previous year, age it forward by one year, and apply the forecasts for fertility, mortality and migration to update the population numbers:

$$
P O P_{t+1}=L_{t} \cdot P O P_{t}+M I G_{t} \cdot P O P_{t}
$$

where POP is an $n \times 1$ vector of gender-specific population totals calculated by age for $n$ ages, $M I G$ is an $n \times 1$ vector of net migration rates by age, and $L$ is the $n \times n$ Leslie Matrix, expressed as follows:

$$
L_{t}=\left[\begin{array}{lllllr}
0 & 0 & \cdots & \mathrm{f}_{\mathrm{x}, \mathrm{t}} & \cdots & \\
1-\mathrm{m}_{0, \mathrm{t}} & 0 & & \cdots & & 0 \\
0 & 1-\mathrm{m}_{1, \mathrm{t}} & 0 & \cdots & & 0 \\
\vdots & & & \ddots & & \\
0 & 0 & \cdots & & & 1-\mathrm{m}_{\mathrm{n}-1, \mathrm{t}}
\end{array}\right]
$$

where $f(x, t)$ and $m(x, t)$ represent the gender specific fertility rates and central death rates for people aged $x$ during the year $t$.

Making a population forecast in this way requires several components: the Japanese population by age and gender in the base year, forecasted fertility rates by-age, the sex ratio at birth, and by-age and by-gender forecasts for mortality and net migration. Our base year for the forecasts is 2006. Our base year population data is for those of Japanese ethnicity, and it is from the Statistics Bureau of Japan's Ministry of Internal Affairs and Communications, as compiled and provided by the Human Mortality Database (2009). Data is available by gender for all ages between 0 and 109, and for the sum of individuals aged 110 and over. In Japan, it is common to use the ethnically Japanese population, rather than the total population in Japan including foreigners, for such matters, and our mortality and fertility data are calculated for the ethnically Japanese population as well. Kaneko et al. (2008) provide forecasts slightly higher than ours, as they consider the total population in Japan including non-Japanese residents. As immigration policy is subject to government 
decisions, the number of foreigners in Japan is not a variable lending itself to stochastic forecasts.

We forecast mortality using the Lee and Carter (1992) method. We use a historical age-sex breakdown of central death rates by single year of age for the years 1947 to 2007, as provided by the Ministry of Health and Welfare's Division of Health and Welfare Statistics through the Human Mortality Database (2009). Call these central death rates $m(x, t)$, where $x$ is age and $t$ is year. The Lee-Carter method uses the notion that mortality can be decomposed into age-specific and time-specific components. To obtain this decomposition, the LeeCarter method considers the relationship:

$$
\ln [m(x, t)]=a(x)+b(x) k(t)+\varepsilon(x, t)
$$

where $a(x)$ represents the time-invariant general age shape across the mortality schedule, and $b(x)$ represents the time-invariant relative speed that various ages respond to the time-varying trend in mortality, which is represented by $k(t)$, and $\varepsilon(x, t)$ represents any remaining residual. This equation implies that mortality rates by age will change at a constant proportion over time, but that the rate of change can differ for different ages. If desired, a second-order can also be estimated by adding the second singular value, which will show any acceleration or deceleration in otherwise linear rate trends. This model looks as follows:

$$
\ln [m(x, t)]=a(x)+b(x) k(t)+c(x) l(t)+\varepsilon(x, t)
$$

where $c(x)$ and $l(t)$ provide the second-order effects of $b(x)$ and $k(t)$, respectively.

In estimating these models, standard regression techniques cannot be used, since none of these terms are observable. Instead, the Lee-Carter method uses the singular-value decomposition (SVD) technique of matrix algebra. ${ }^{1}$ This entails setting $a(x)$ equal to the time average of $\ln [m(x, t)]$ over history, and using a matrix of the deviations, $\ln [m(x, t)-a(x)]$, in the SVD routine. Uniqueness results from using the constraints that the sum of the $k(t)$ terms over $t$ equals zero, and the sum of the $b(x)$ terms over $x$ equals one. To use the SVD method, the data matrix is set with the span of ages along the horizontal axis, and the years along the vertical axis.

A similar procedure is used to forecast fertility, as described in Lee (1993). Fertility data are aggregated from two sources, and are available for the years 1947 to 2006 . First,

\footnotetext{
${ }^{1}$ The theorem of the singular value decomposition states that any $\mathrm{m} \times \mathrm{n}$ matrix can be factored into $\mathbf{X}=\mathbf{U} \times \mathbf{S} \times \mathbf{V}^{\prime}$, where $\mathrm{U}$ is an $\mathrm{m} \times \mathrm{m}$ orthogonal matrix, $\mathrm{V}$ is an $\mathrm{n} \times \mathrm{n}$ orthogonal matrix, and $\mathrm{S}$ is an $\mathrm{m} \times \mathrm{n}$ matrix with the singular values of $\mathrm{X}$ on the main diagonal, and zeros elsewhere. The singular values are non-negative and are typically ordered from largest to smallest along the main diagonal of S.
} 
Japan's Bureau of Statistics provides annual data on fertility rates by five-year age groups (15-19, 20-24, 25-29, 30-34, 35-39, 40-44, and 45-49) for each year between 1947 and 2006. Second, NIPSSR (2008) provides fertility rates for each age between 15 and 49, for 1947 and 2006, as well as for five-year intervals between 1950 and 2005. We mix and expand these datasets together to get fertility rates for each age and for each year between 1947 and 2006.

The equation now is:

$$
f(x, t)=a(x)+b(x) f(t)+\varepsilon(x, t)
$$

where $f(x, t)$ represents the fertility rates for mothers of age $x$ in year $t$. To ensure a unique solution, we again use the same constraints on the $b(x)$ and $f(t)$ terms. Because fertility rates tend to be behave more randomly, Lee (1993) adds a long-term constraint around which the total fertility rate fluctuates and provides absolute bounds to prevent fertility from falling below zero.

After making these decompositions, we use ARIMA time series models to estimate and forecast the time varying trends in mortality and fertility. The key to forecasting these age-sex variables is to assume that the age-specific components of the model will remain constant in the future, and to fit a time series model to the time components. After fitting an appropriate model, 2,500 Monte Carlo simulations are produced using the specified model, in order to obtain a probability distribution over the time-specific components of mortality and fertility. For each simulation, the process of calculating the associated central death rates and fertility rates is a straightforward application of equation (3), (4), or (5). Mortality and fertility are not correlated as is consistent with the stochastic forecasting literature described in Keilman, Pham, and Hetland (2002).

In the case of net migration for ethnically Japanese people, we use deterministic assumptions based on historical data, rather than attempting a stochastic forecast. This is because migration plays a relatively small role in population changes for Japan, and because obtaining detailed historical data by-age is difficult. Though some studies have included stochastic migration forecasts, it is still common to simply include migration with deterministic assumptions. Our net migration data are for the ethnically Japanese population and are from Kaneko et al. (2008). These data represent the average value of age and sexspecific net migration rates between 1995 and 2005, excluding 2001-2004 for geopolitical reasons, and then smoothing the data.

\section{Mortality Forecasts}


Figure 1 shows historical mortality rates and their logarithms by gender for selected ages. The figure demonstrates a general trend in decreasing mortality over time for all ages and fairly constant age-specific rates of decline. In particular, infant mortality declines much more rapidly than mortality at other ages.

\section{// Figure 1 About Here //}

The decomposition of the time and age-specific components can be seen in Figure 2. For both genders, the $a(x)$ term shows that mortality rates at very young ages tend to be high, but decline rapidly during childhood, and then rise consistently into adulthood and old age. The pattern for the $b(x)$ term for both genders is also similar, and it shows the speed at which mortality responds to the time component for different ages. With this term, we see that mortality is declining most rapidly at young ages, and the decline continues, though at a slower pace, for older ages. Finally, the $c(x)$ term tends to be negative at younger ages, meaning that the rate of decline is slowing down, but is positive at older ages, meaning that while we saw slower decreases in mortality for these ages, we can now see that the rate of decrease is increasing at the older ages.

\section{// Figure 2 About Here //}

Figure 3 compares the historical data with the fitted estimates from the first-order model for the historical logged male mortality rates for the same four ages shown in Figure 1. We can observe that the parsimonious first-order model fits the historical data quite well, as it is able to match the different by-age trends.

\section{// Figure 3 About Here //}

We use a first-order model for the logarithms of mortality rates, and we find that the time component is stationary for both males and females at the five percent level. For males, we find an AR(1) model is most appropriate when testing for significance of the final lag at five percent, starting with a maximum lag length of eight. Meanwhile, we find an AR(3) model fits best for females. With these fitted models of the time component, we make 2,500 stochastic simulations through 2105. Using these simulations, we are able to calculate 2,500 scenarios for the future evolution of mortality rates by age and gender. Figure 4 shows the results of these stochastic forecasts for male mortality at four different ages, in order to demonstrate the general trends in our results. We find that the rates of mortality decline have slowed and that the 95 percent confidence intervals appear small compared to past reductions in mortality. 
A better summary of the stochastic forecasts for mortality is period life expectancies, which represent the number of years in which the probability of remaining alive is above 50 percent using the by-age mortality rates of a given year. Figure 5 shows both the historical data and the stochastic forecasts with the median and 95 percent confidence interval. The figure also provides the three alternative scenarios for life expectancy provided by NIPSSR in Kaneko et al. (2008). We can observe a continuing increase in life expectancies as mortality continues to decline, but the rate of increase will be slower than in the past. In this regard, our forecasts show higher mortality rates, and thus lower life expectancies, than do the forecasts of NIPSSR, as the 97.5 percentile of our forecasts match closely only with their most pessimistic forecasts. The other feature to note in the comparison is that our confidence interval for male life expectancies is wider than the NIPSSR projections, while our confidence interval for females is narrower.

\section{// Figure 5 About Here //}

\section{Fertility Forecasts}

As shown in Figure 6, the historical fertility rates for Japan show that declining fertility rates are not universal at all ages. Rather, women are tending to delay childbirth until later. Thus, while the fertility rates of 25 year olds have fallen quite dramatically, the decline is much slower for 30 year olds, and since 1980 there has been an upward trend in fertility for 35 year old women.

\section{// Figure 6 About Here //}

When considering fertility, we must consider the birth ratio for dividing births between genders. Between 1980 and 2006, the average number of males born relative to females was 1.0558. It means that 51.36 percent of new babies are male. This number does not fluctuate much since 1980, and we assume this proportion to stay constant in the future.

The time and age-specific components for fertility are shown in Figure 7. The time component shows that fertility has been declining steadily since about 1970 . Meanwhile, the $a(x)$ term shows that fertility rates rise rapidly through the teens and twenties and peak in the late 20s before declining at subsequent ages. The $b(x)$ term shows the speed of response to the declining time component. Fertility rates are declining rapidly for women in their 20s but are relatively flat at later ages. We estimate a first order model on the levels data for fertility rates. A unit root test shows that the time component for fertility is nonstationary at the five percent level, and there is no clear trend to model, but in order to include a long-term constraint for the total fertility rate as suggested in Lee (1993), we estimate an AR(1) model in which total fertility is bound above by four babies per woman, bound below by zero babies 
per woman, and fluctuates around a long-run average of 1.26 babies per woman, which matches the assumption in Kaneko et al. (2008). After estimating this model, we again create 2,500 stochastic simulations through 2105.

\section{// Figure 7 About Here //}

The total fertility rate forecast shown in Figure 8 summarizes the combined effects of the fertility rates at different ages, as it is the sum of fertility rates across ages in a given year. Because of our included constraint, the median long-term forecast is about 1.26. The 95 percent confidence interval for the stochastic forecasts is substantially larger than the three scenario deterministic forecasts, as historical volatility in fertility suggests greater potential for fertility to rebound than Kaneko et al. (2008) assume. By 2055, the long-term trends are stabilized, and the 97.5 percentile is 1.92 babies per women, which is slightly less than the population replacement rate, while our 2.5 percentile is 0.83 babies per woman. This range is 86 percent of the median forecast size. In contrast, the range provided by Kaneko et al. (2008) is 1.06 to 1.55 , which is only 31 percent of their medium forecast size. Even with past volatility considered, our simulations do suggest it is highly unlikely for fertility in Japan to return to the replacement rate level during the next 100 years.

\section{// Figure 8 About Here //}

\section{Net Migration Forecasts}

As explained, we do not make a stochastic forecast for net migration. Figure 9 shows the forecast to be applied in all future years. For both genders, there is a small net outflow of Japanese people from Japan, but the outflows are only a fraction of a percent. By their mid30s, women are returning to Japan, though there is not a net inflow of Japanese men until after age 65.

\section{// Figure 9 About Here //}

\section{Stochastic Forecast for the Japanese Population}

Using the iterative process with the Leslie matrix, we are able to combine the above forecasts with the base year population to create a stochastic forecast of Japan's population. Figure 10 shows the results for the total population of Japan, including the historical data, the median and 95 percent confidence interval for the stochastic simulations, and the three deterministic scenarios of Kaneko et al. (2008). As mentioned, the population in Kaneko et al. (2008) includes foreigners and so tends to be several million people larger than our forecasts, but otherwise, the forecasts are fairly similar, except that the stochastic forecasts show greater variability than the deterministic forecasts. Japan's population can almost surely be expected to decline in the coming years. In 2055, the median stochastic forecast is 
86.86 million people, and the confidence interval width is 26 percent of the median size, while the medium deterministic forecast is 92.01 million people with a forecast range that is 18 percent of this size. By 2105, our median projection indicates only 41.46 million people of Japanese ethnicity with a confidence interval spanning 101 percent of this size, while the deterministic medium projection is 45.8 million people with a range of estimates 63 percent of this size. How this total population is divided into different age groups will have important implications for budget sustainability.

\section{// Figure 10 About Here //}

Figure 11 shows Japan's population pyramid in the base year, 2006, as well as the stochastic simulations of the median surrounded by the 95 percent confidence interval for the population pyramid in 2050 and 2105. Particularly in 2050, we can see the important role of fertility in the forecasts, as older people were already born in the base year and the small interval width is explained only by differences in future mortality, while younger people were born after the simulations began and so the variation in their populations depends both on the variation of mortality and fertility. The other feature to note is that by 2006, the term “pyramid” is no longer descriptive of Japan's population structure, and this will almost surely continue to be the case in subsequent years as fertility stays below the replacement rate.

\section{// Figure 11 About Here //}

Next, Figure 12 provides forecasts for different age groups as a percentage of the total population. These include the median stochastic forecast surrounded by the 95 percent confidence interval, as well as five deterministic forecasts from Kaneko et al. (2008). As described earlier, the most extreme deterministic forecasts for different age groups as a percentage of the population are different from the forecasts that produce the most extreme total populations. Nonetheless, as depicted in the figure, the stochastic forecasts remain more volatile than the deterministic forecasts, and the median simulation shows that about 40 percent of people in Japan will be elderly by 2105. The importance of noting these wider intervals is that policymakers will need to be prepared to confront a wider variety of situations in the future as they seek to provide suitable and sustainable pension systems.

\section{// Figure 12 About Here //}

Figures 13 and 14 provide stochastic simulations of the different age groups together so as to make more direct comparisons, first as a percentage of the total population, and then as the absolute numbers of people. Figure 14, in particular, reveals that the number of working-age people will continue to fall very rapidly in the coming years as more people are reaching the retirement age than are being born, but the number of elderly people in Japan 
will continue growing until about 2020 and then remain stable until about 2040 before gradually falling afterward. In terms of the old-age dependency ratio, which is the number of elderly people divided by the number of working age people, our median results indicate that in 2007 there is one elderly for every three working-age people, in 2030 there are slightly more than one elderly for every two working-age people, and after 2050 the ratio settles at about three elderly for every four working-age people. The range of the confidence interval in 2105 extends from 0.52 elderly per working-age person to 1.12 elderly per working-age person. Though the range is wide, such a ratio will place a huge burden on the working-age population and is unprecedented in world history.

\section{// Figure 13 About Here // \\ // Figure 14 About Here //}

Finally, Figure 15 provides a probability distribution for the year that Japan's population falls to 100 million people. Our median forecast suggests this will happen in 2043, with a 95 percent confidence interval ranging from 2038 to 2056. In contrast, the deterministic forecast suggests a smaller range of possibilities, from 2041 to 2055, with a medium guess of 2046. Policymakers must be prepared to deal with a rapidly decreasing Japanese population in the coming years.

\section{// Figure 15 About Here //}

\section{CONCLUSIONS}

We find that Japan's population can be expected to fall in the coming years, and that the potential range of future population levels is significantly wider than found in the deterministic forecasting approach used by the NIPSSR. In subsequent research, we hope to include a stochastic forecast for net migration in order to determine how much impact this may have on the projections, and we also hope to use these forecasts in order to study pension reform and government budgeting issues in Japan in the context of intergenerational equity.

\section{ACKNOWLEDGEMENTS}

We wish to thank Dr. Reiko Hayashi (President, Linz Co., Ltd, Tokyo, Japan) for her assistance in finding data sources for the paper. Funding for this research was provided by the Japan Society for the Promotion of Science (JSPS) Grants-in-Aid \#18530198. 


\section{REFERENCES}

Advisory Council on Social Security (1991), The Social Security Technical Panel Report to the 1991 Advisory Council on Social Security, Washington, D.C., Advisory Council on Social Security.

Board of Trustees of the Federal Old-Age and Survivors Insurance and Disability Trust Funds (2003), 2003 Annual Report, Washington, D.C., US Government Printing Office.

Børlum, M. (2004), "Demographic Uncertainty and the Sustainability of the Danish Pension System - A Stochastic Approach,” Centre for Economic and Business Research Working Paper 2004-03, Frederiksberg, Denmark, Copenhagen Business School.

Burdick, C. and J. Manchester (2003), "Stochastic Models of the Social Security Trust Funds,” Social Security Bulletin 65(1), pp.26-31.

Congressional Budget Office (2005), Quantifying Uncertainty in the Analysis of Long-Term Social Security Projections, Washington, D.C., Congressional Budget Office.

Fehr, H. and C. Habermann (2004), "Pension Reform and Demographic Uncertainty: The Case of Germany," Paper Written with the EU-Project "Demographic Uncertainty and the Sustainability of Social Welfare Systems," Wuerzburg, Germany, University of Wuerzburg.

Giang, L. T. and W. D. Pfau (2009), "Demographic Changes and the Long-Term Pension Finances in Vietnam: A Stochastic Actuarial Assessment," Journal of Population Ageing 1(2), forthcoming.

Human Mortality Database (2009), University of California, Berkeley (USA), and Max Planck Institute for Demographic Research (Germany), downloaded on 21 April 2009 URL: www.mortality.org

Kaneko, R., A. Ishikawa, F. Ishii, T. Sasai, M. Iwasawa, F. Mita, and R. Moriizumi (2008), "Population Projections for Japan 2006-2055: Outline of Results, Methods, and Assumptions,” The Japanese Journal of Population 6(1), pp.76-114.

Hyndman, R. J. and H. Booth (2008), "Stochastic Population Forecasts Using Function Data Models for Mortality, Fertility, and Migration,” International Journal of Forecasting 24, pp.323-342.

Keilman, N., D. Q. Pham, and A. Hetland (2002), "Why Population Forecasts Should be Probabilistic - Illustrated by the Case of Norway,” Demographic Research 6, pp.409454.

Kitamura, T., H. Tsurubuchi, and K. Nakashima (2006), “Application of Stochastic Alm Model for Public Employees' Pension in Japan - Which Indexation Rule is Appropriate, Maintaining Nominal Pension Amount or Not?” Social Science Research Network Working Paper. Available at SSRN: http://ssrn.com/abstract=891199, accessed May 7, 2009.

Kunieda, S. (2002), “Japanese Pension Reform: Can We Get Out of Intergenerational Exploitation?” Hitotsubashi Journal of Economics 43, pp.57-71. 
Lee, R. D. (1993), "Modeling and Forecasting the Time Series of US Fertility: Age Distribution, Range, and Ultimate Level," International Journal of Forecasting 9, pp.187-202.

Lee, R. D. (1999), “Probabilistic Approaches to Population Forecasting,” Population and Development Review, supp to Vol. 24, pp.156-190.

Lee, R. D. and L. R. Carter (1992), “Modeling and Forecasting U.S. Mortality,” Journal of the American Statistical Association 87(109), pp.659-671.

Lee, R. D., M. W. Anderson, and S. Tuljapurkar (2003), "Stochastic Forecasts of the Social Security Trust Fund," Report Prepared for the Social Security Administration, Washington, D.C., Social Security Administration.

Lee, R. D. and S. Tuljapurkar (1994), "Stochastic Population Forecasts for the United States: Beyond High, Medium, and Low," Journal of the American Statistical Association 89(428), pp.1175-1189.

Lee, R. and S. Tuljapurkar (2001), "Population Forecasting for Fiscal Planning: Issues and Innovations,” in: A.J. Auerbach and R. Lee, eds., Demographic Change and Fiscal Policy, Cambridge, Cambridge University Press, pp.7-57.

National Institute of Population and Social Security Research (various years), Population Statistics of Japan, Tokyo, NIPSSR.

Pfau, W. D. (2003), Essays on Social Security Reform, Unpublished Ph.D. Dissertation, Department of Economics, Princeton University (USA).

Pfau, W. D. and V. Atisophon (2009), "The Impact of the National Pension Fund on the Suitability of Elderly Pensions in Thailand,” Asian Economic Journal 23(1), pp. 41-63.

Sabelhaus, J. and J. Topoleski (2007), "Uncertain Policy for an Uncertain World: The Case of Social Security,” Journal of Policy Analysis and Management 26(3), pp.507-525.

Suzuki, W., M. Yuda, and K. Kawasaki (2003), “Jinko Yosoku no Fukakujitsusei to Nenkin Zaisei: Monte Carlo Simulation o Mochiita Jinko Yosoku no Shinrai Kukan Sanshutsu to Nenkn Zaisei eno Eikyo [Uncertainty of Population Forecast and Pension Financing - Computation of Confidence Intervals for Population Forecast Using Monte Carlo Simulation and Its Implication on Pension Budget Balance],” Kaikei Kensakenku [Audit Inspection Research] 28, pp.101-112.

Tuljapurkar, S. (2006), "Population Forecasts, Fiscal Policy, and Risk,” Paper for Conference on "Government Spending on the Elderly," Annandale-on-Hudson, NY, Levy Economics Institute at Bard College.

Yamashige, S. (2007), "Declining Fertility: Theories and Japanese Experience." Paper Presented at the Hitotsubashi University International Symposium on Social Policy in Asia, Tokyo, Hitotsubashi University. 
Figure 1: Mortality Rates in Japan, 1947-2007
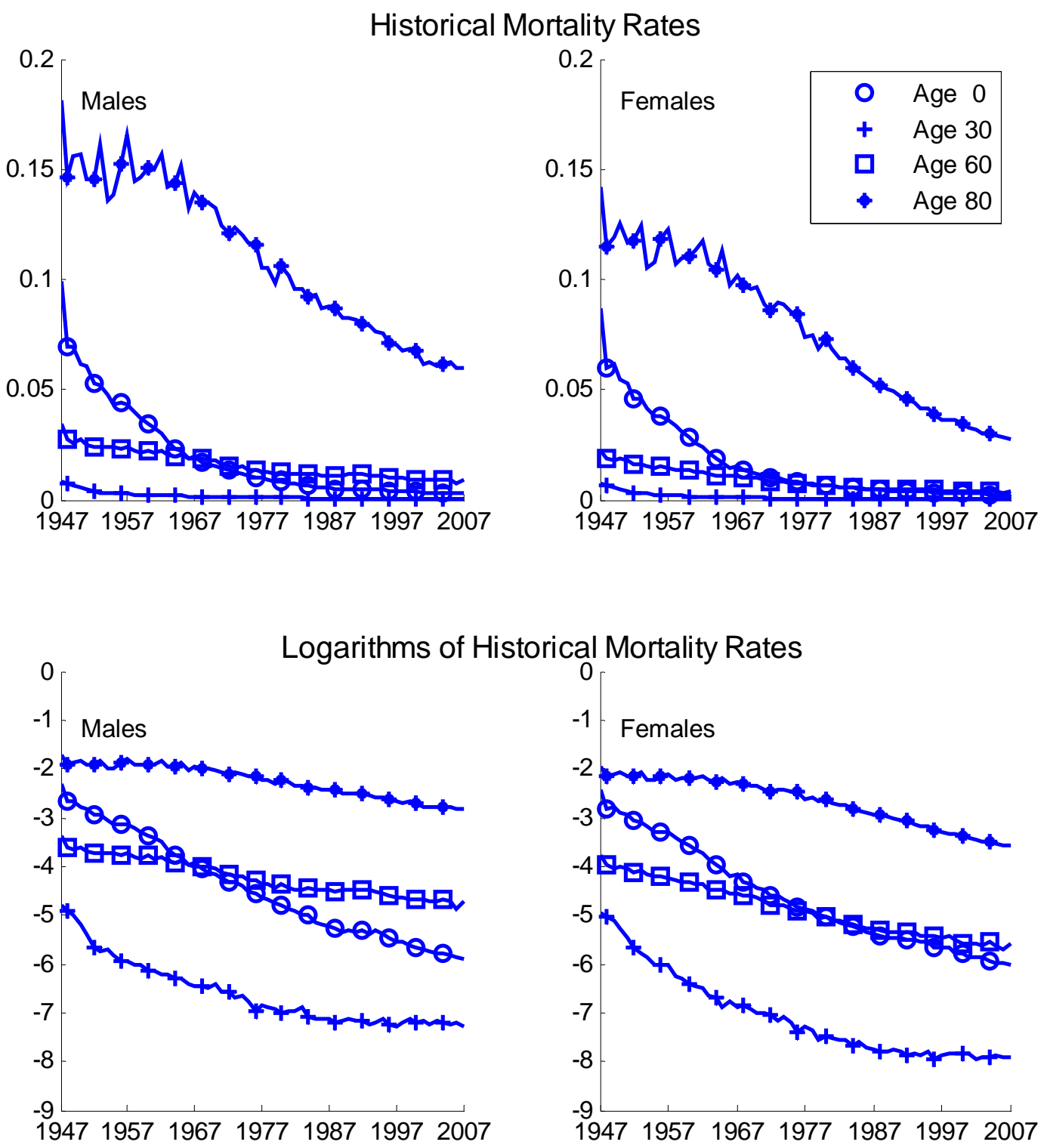

Data Source: Japan's Ministry of Health and Welfare, Health and Welfare Statistics and Information Department, as compiled and provided by Human Morality Database (2009). 
Figure 2: Time and Age-Specific Components for the Mortality Model
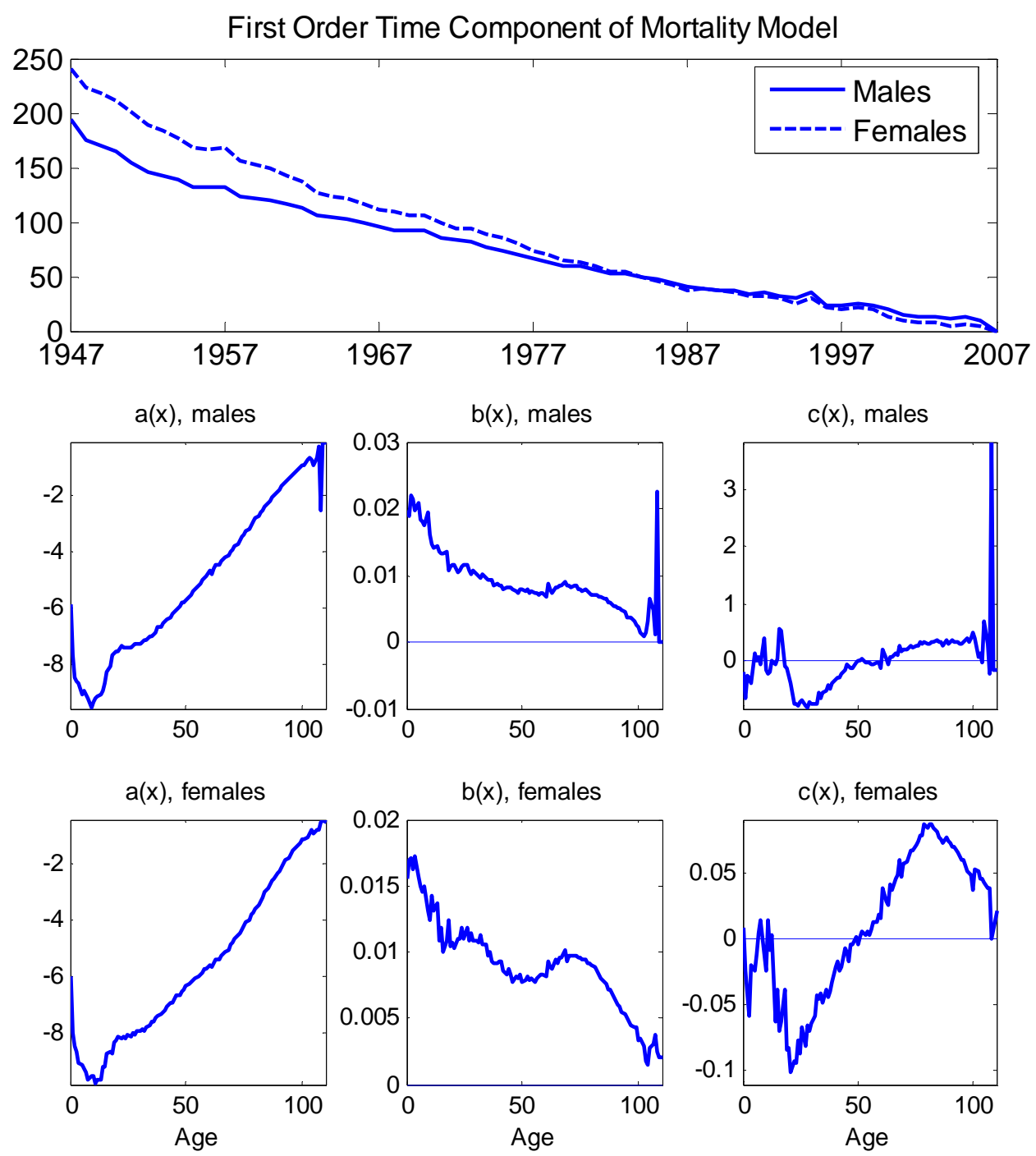

Data Source: See Figure 1. 
Figure 3: Comparison of Model Estimates to Logged Mortality Rates for Males, 1947-2007

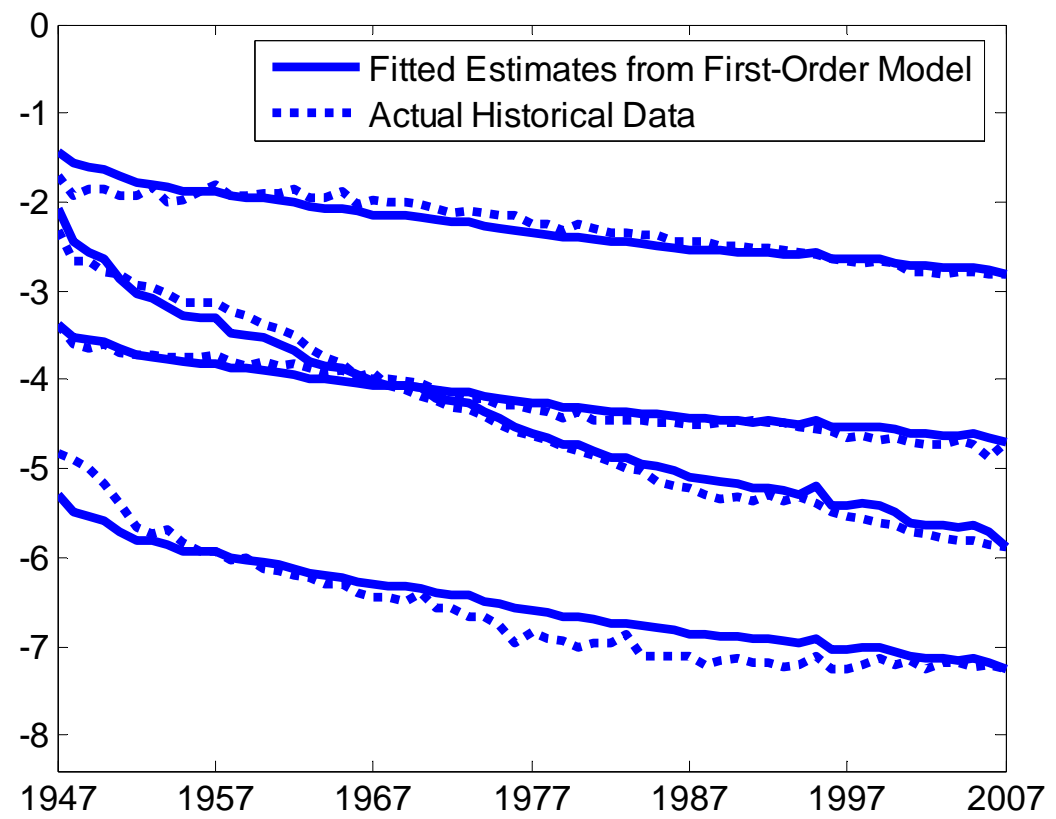

Data Source: See Figure 1.

Figure 4: Historical Data and Stochastic Simulations (Median and 95 Percent Confidence Interval) for Male Mortality
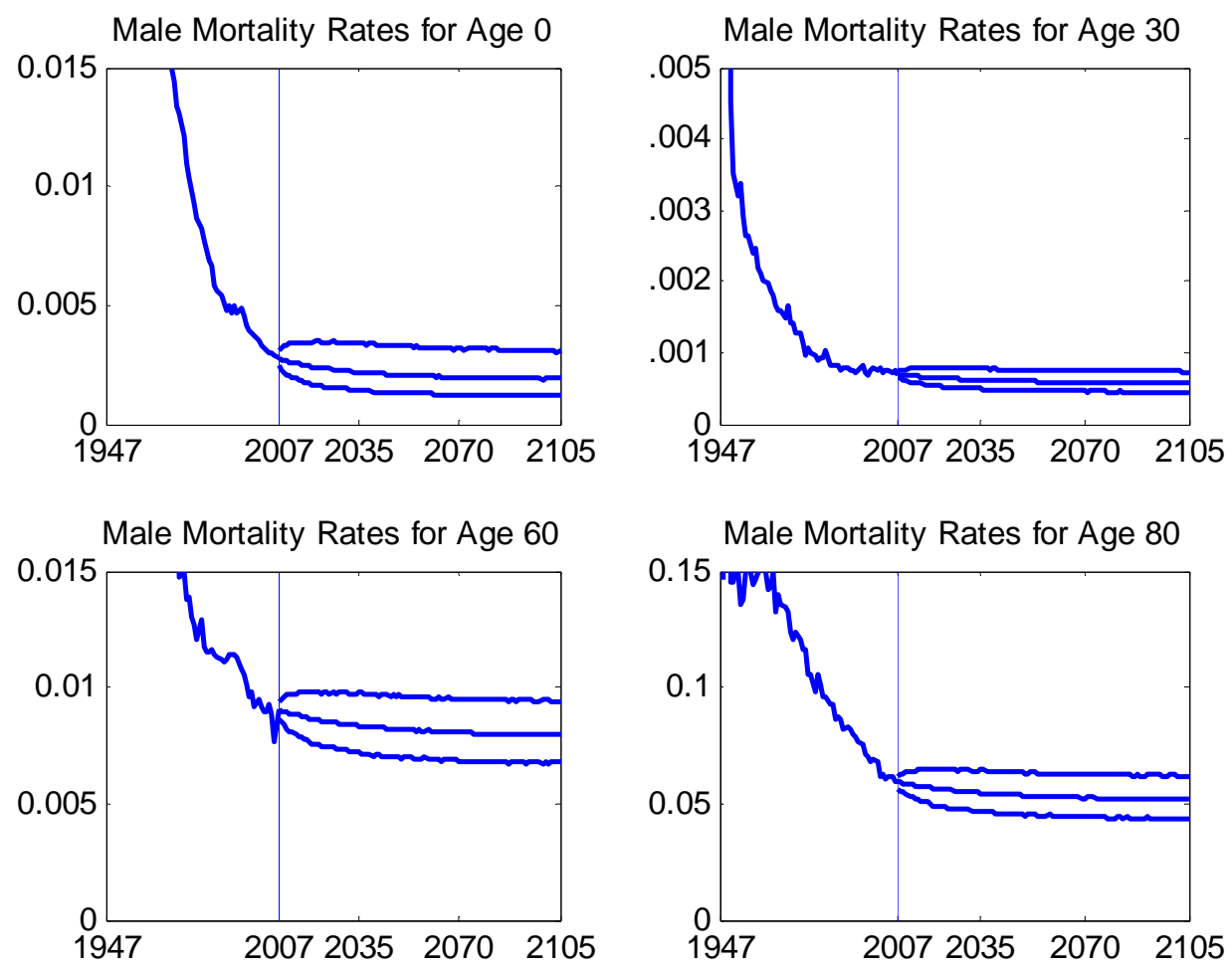

Data Source: See Figure 1. 
Figure 5: Historical Data, Stochastic Simulations (Median and 95 Percent Confidence Interval), and Deterministic Forecasts for Period Life Expectancies at Birth
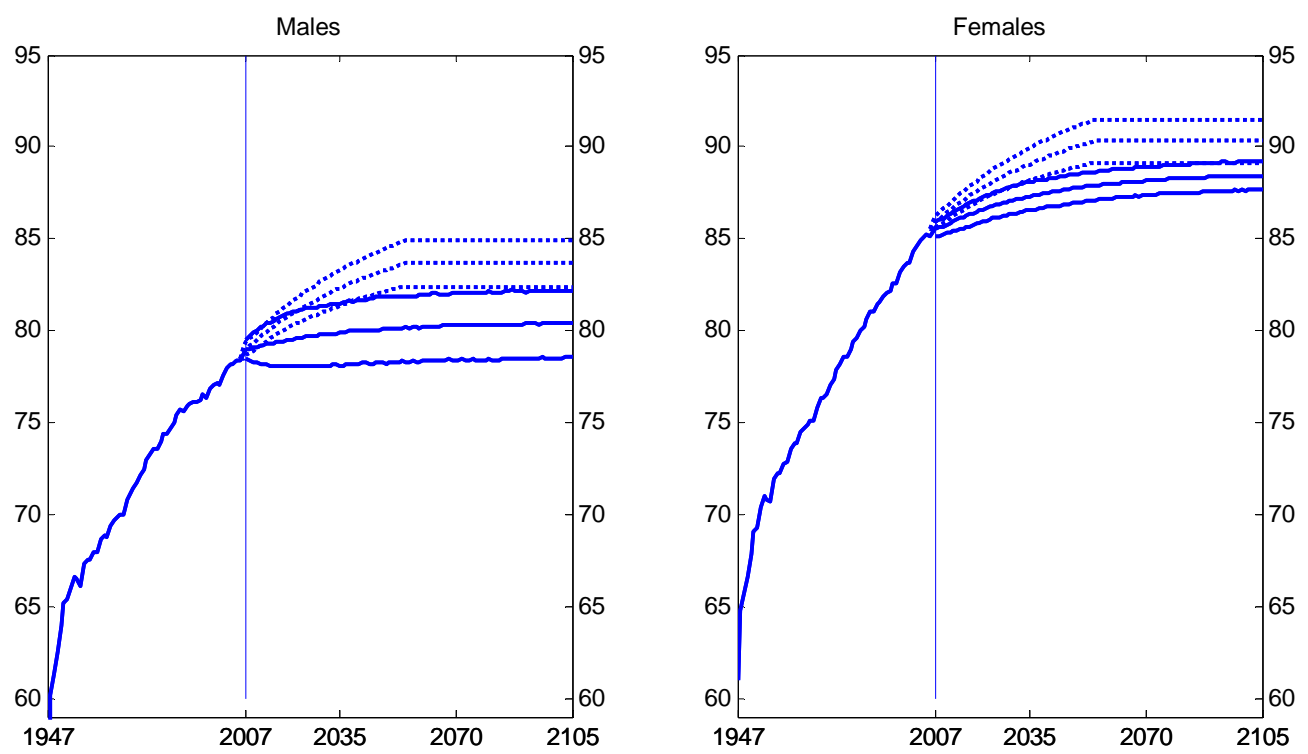

Data Source: See Figure 1.

Figure 6: Fertility Rates in Japan, 1947-2006

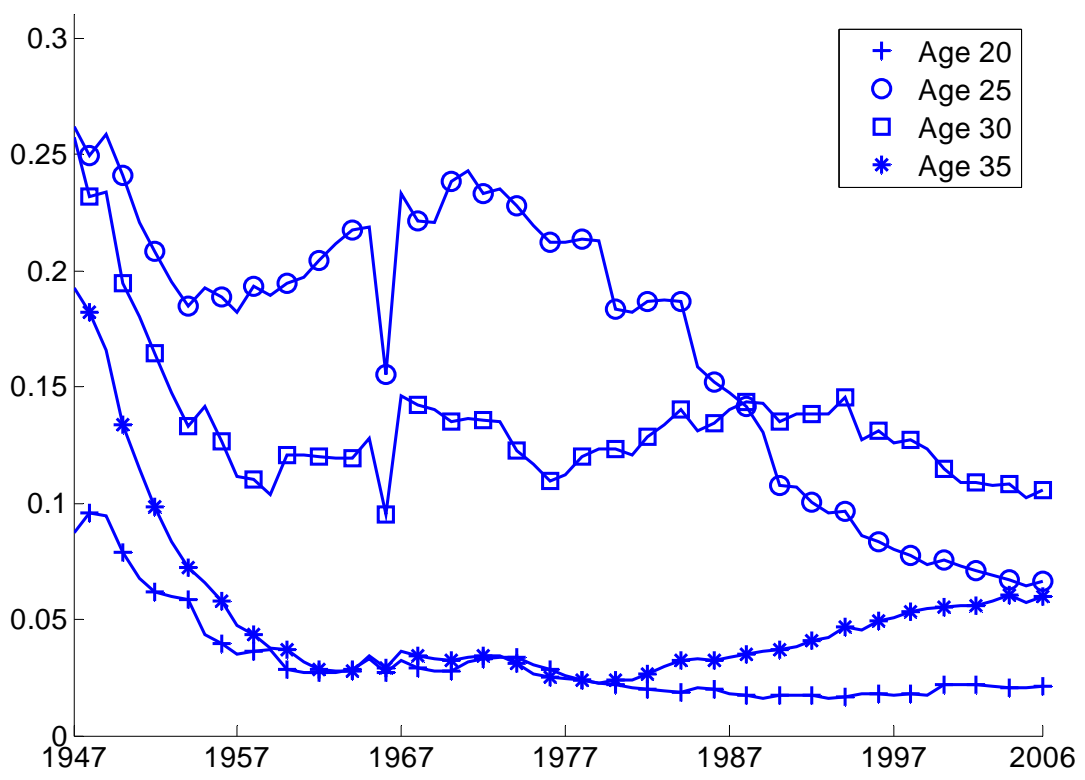

Data Source: Japan's Bureau of Statistics and National Institute of Social Security and Population Research (2008). See text for further explanation. 
Figure 7: Time and Age-Specific Components for the Fertility Model

First Order Time Component of Fertility Model
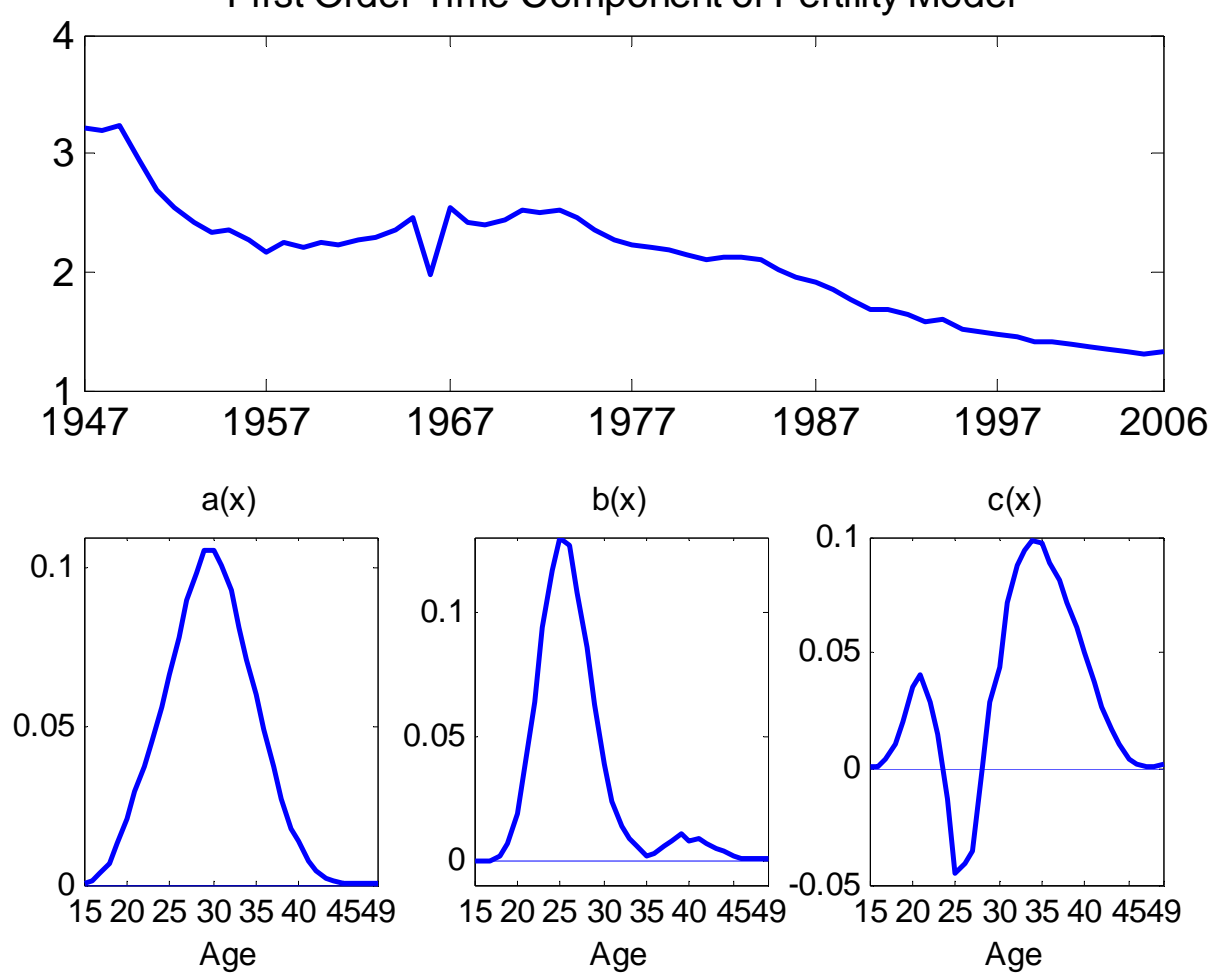

Data Source: See Figure 6.

Figure 8: Historical Data, Stochastic Simulations (Median and 95 Percent Confidence Interval), and Deterministic Forecasts for Total Fertility Rates (TFR)

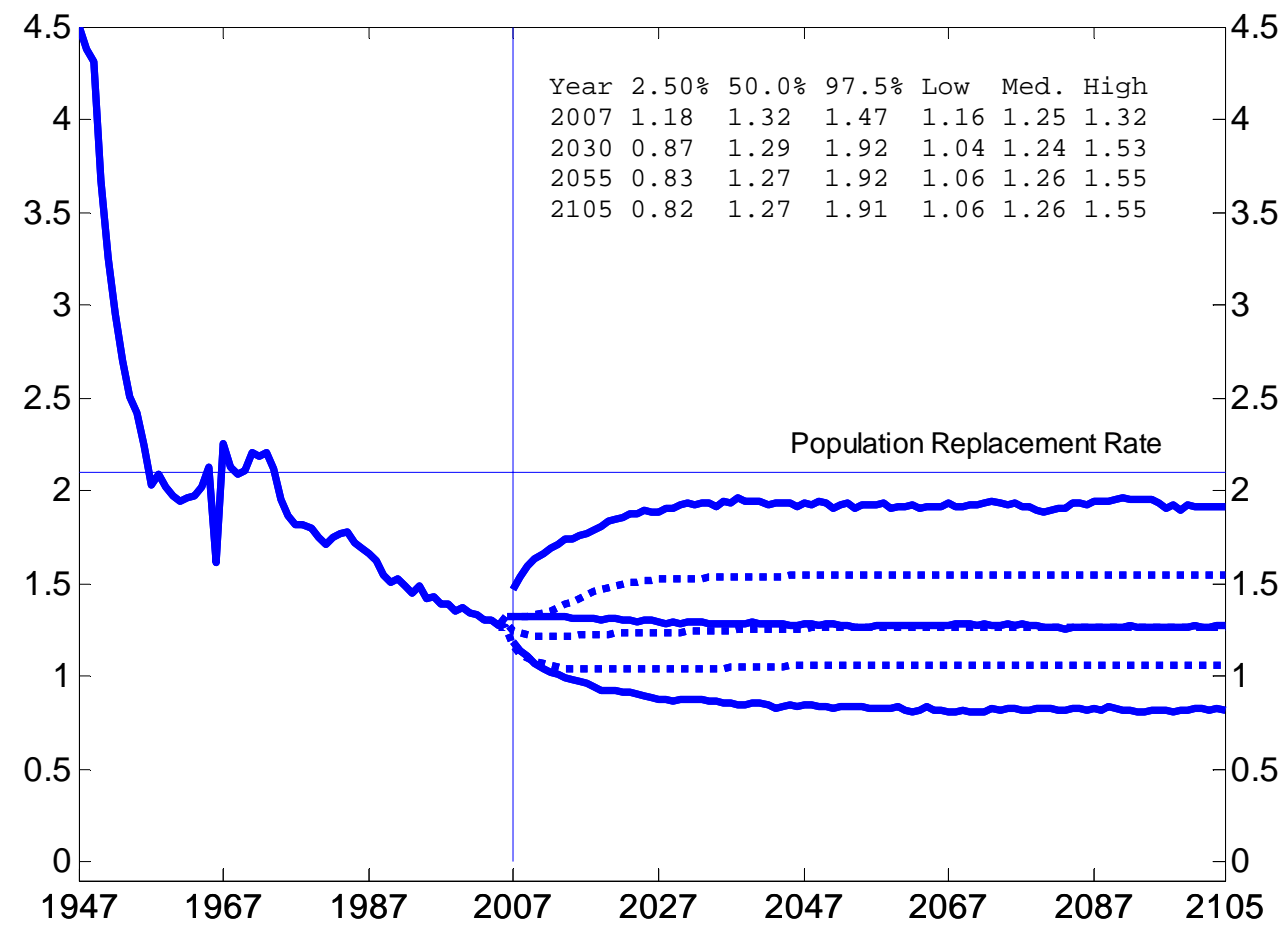

Data Source: See Figure 6. 
Figure 9: Net Migration Rates of Japan's Population by Age and Gender

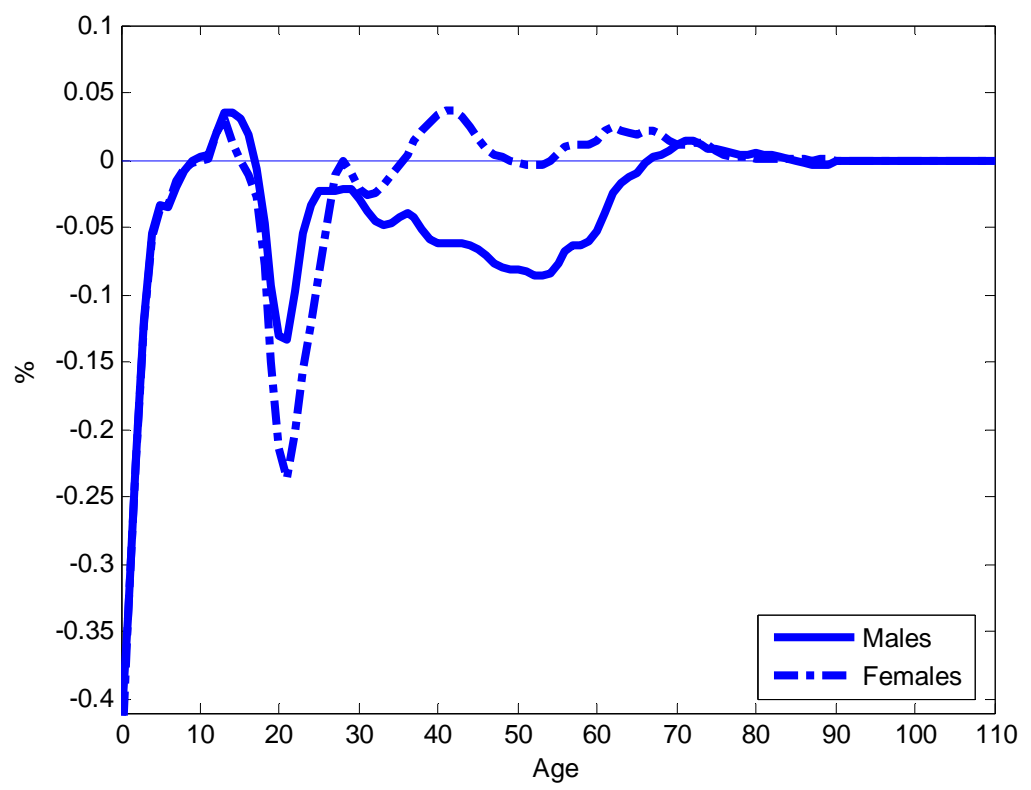

Data Source: Kaneko et al. (2008). See text for further explanation.

Figure 10: Historical Data, Stochastic Simulations (Median and 95 Percent Confidence Interval), and Deterministic Forecasts for Total Population of Japan, 1947-2105

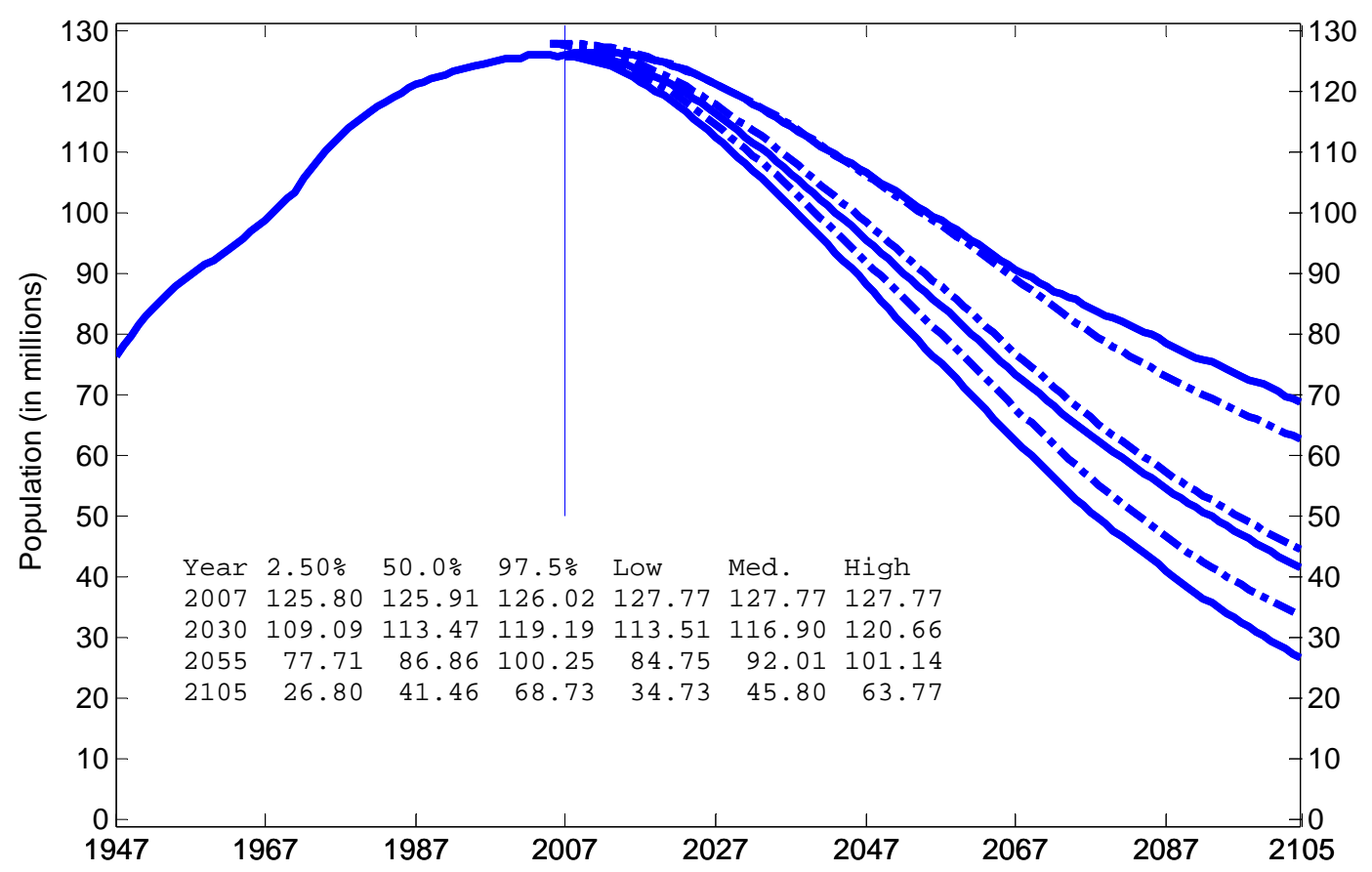

Data Source: Total population data for Japan is from the Statistics Bureau of Japan's Ministry of Internal Affairs and Communications, as compiled and provided by Human Morality Database (2009). Our estimates use previously described sources for mortality, fertility, and net migration as well. Deterministic forecasts are provided in Kaneko et al. (2008). 
Figure 11: Stochastic Simulations (Median and 95 Percent Confidence Interval) For Japan's Population Pyramid in Selected Years
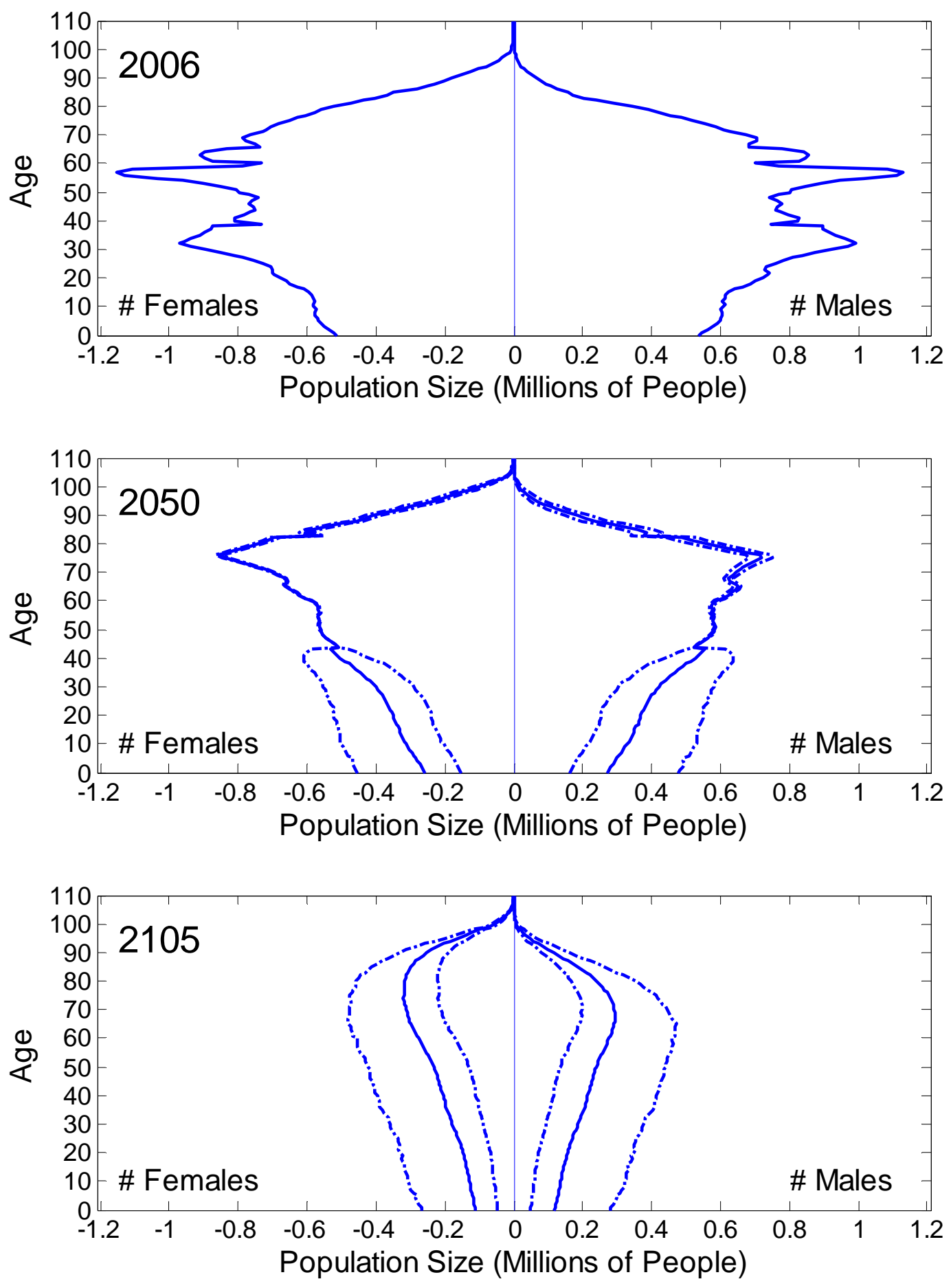

Data Source: See Figure 10. 
Figure 12: Historical Data, Stochastic Simulations (Median and 95 Percent Confidence Interval), and Deterministic Forecasts for Percentage of Population by Age Group in Japan, 1947-2105
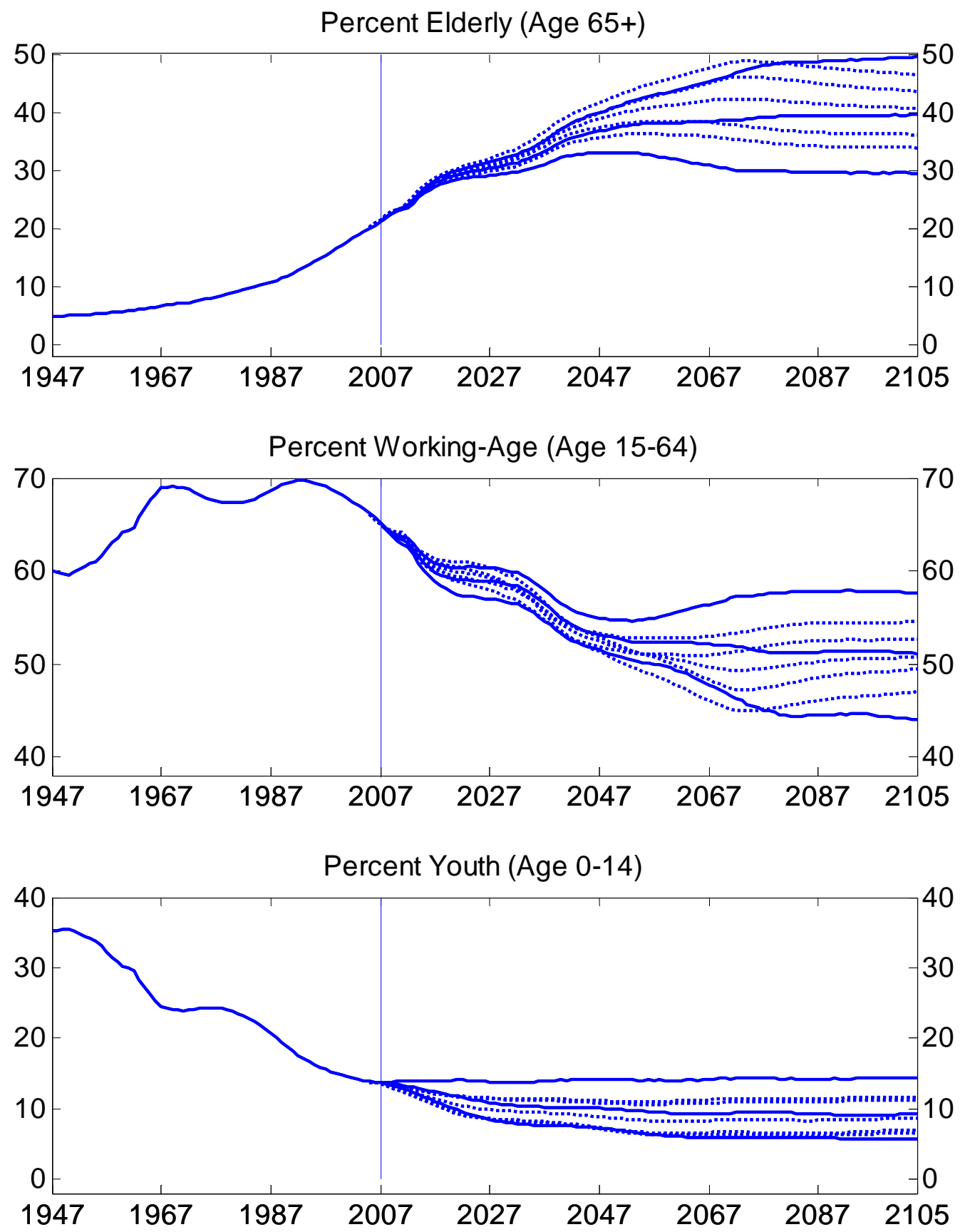

Data Source: See Figure 10. 
Figure 13: Historical Data and Stochastic Simulations (Median and 95 Percent Confidence Interval) for Percentage of Population by Age Group in Japan, 1947-2105

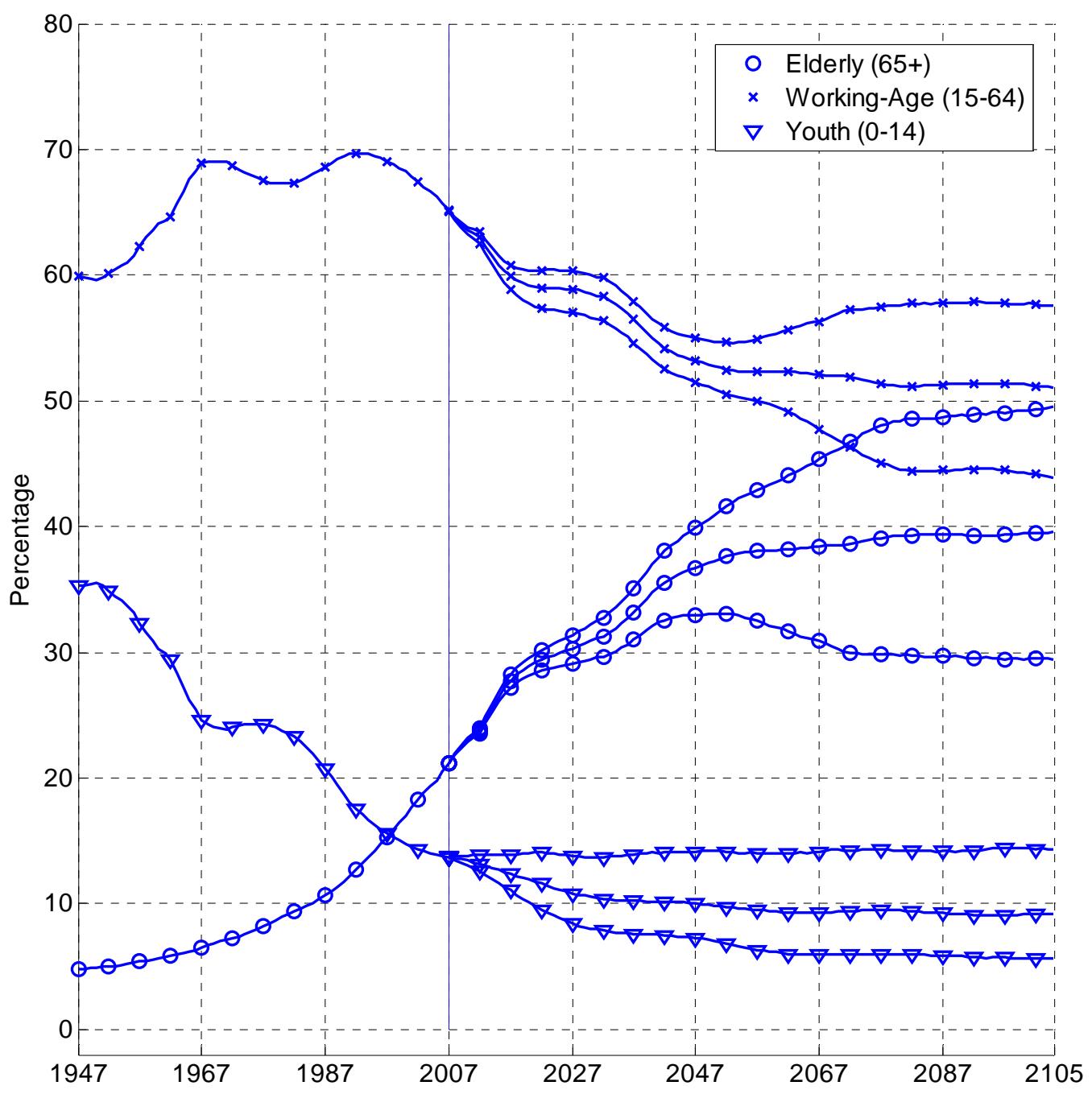

Data Source: See Figure 10. 
Figure 14: Historical Data and Stochastic Simulations (Median and 95 Percent Confidence Interval) for Total Population by Age Group in Japan, 1947-2105

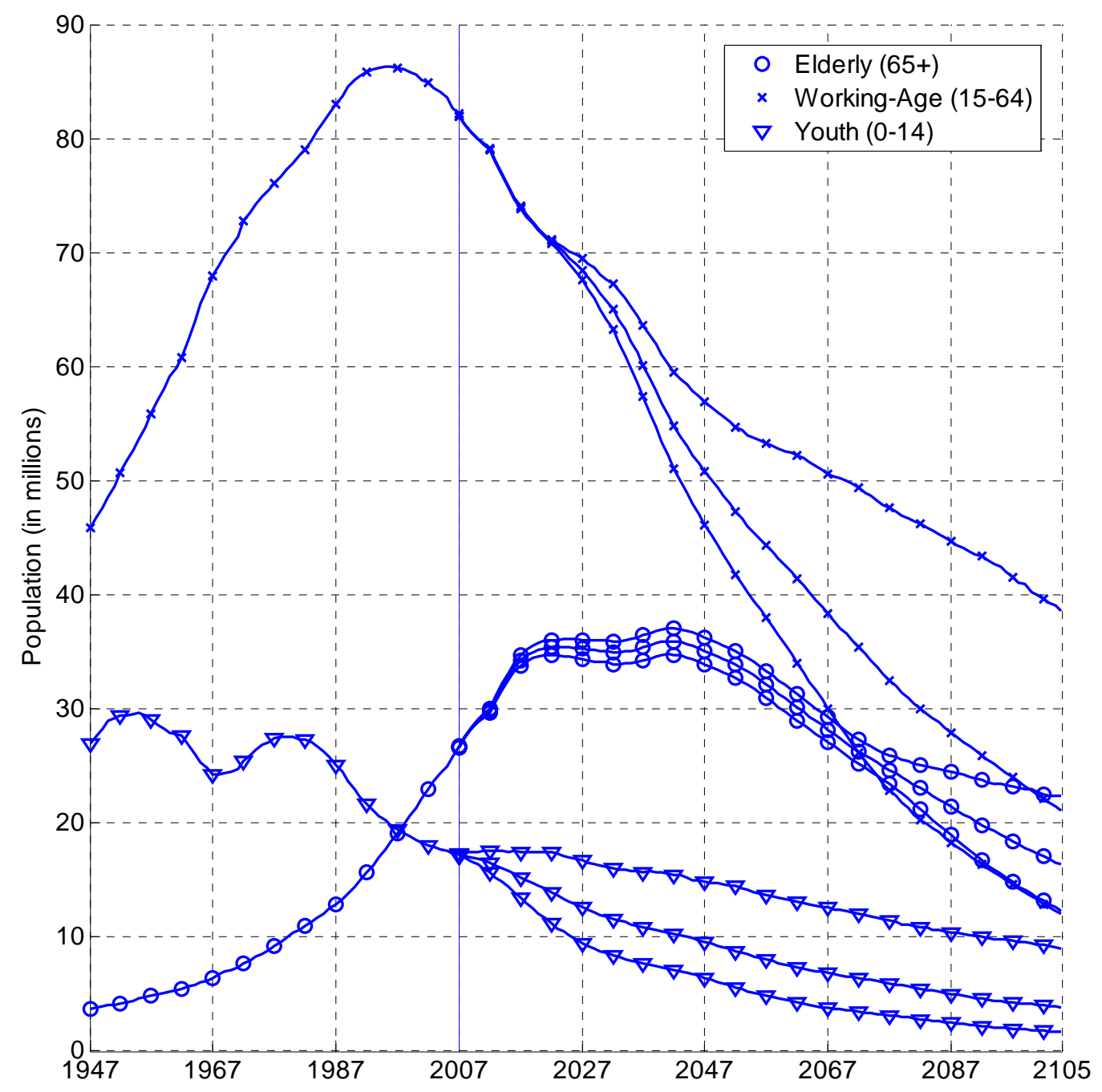

Data Source: See Figure 10. 
Figure 15: Simulated Distribution for When Japan's Population Falls to 100 Million

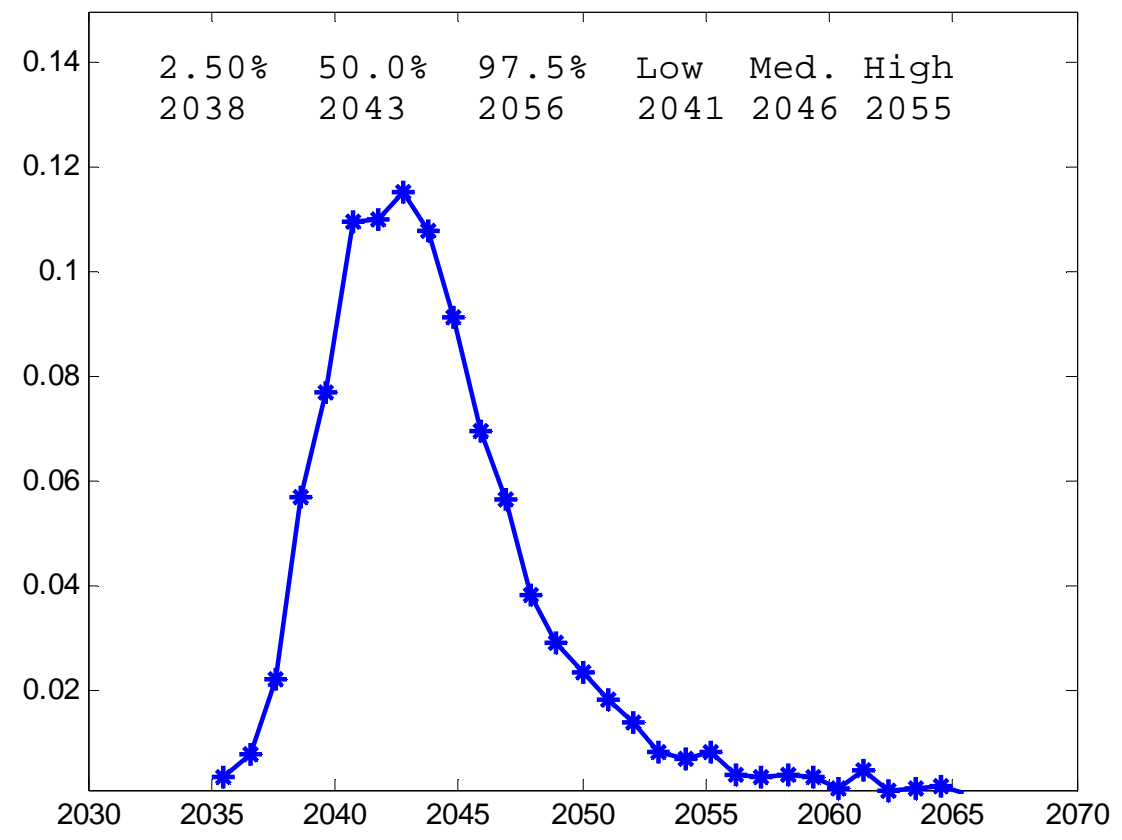

Data Source: See Figure 10. 